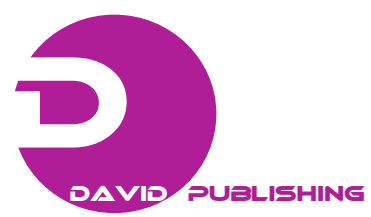

\title{
Antigenic Variation of Capsid Protein Vp1 of Foot and Mouth Disease Virus Prevalent in South Asian Regions
}

\author{
S.M. Sabbir Alam*, Md. Ruhul Amin, Munawar Sultana and Md. Anwar Hossain \\ Department of Microbiology, University of Dhaka, Dhaka 1000, Bangladesh \\ *Corresponding author's e-mail: sabbir.alam.mb@gmail.com
}

\begin{abstract}
Foot and mouth disease virus (FMDV) includes seven distinct serotypes and is highly contagious infecting mainly cloven-hoofed animals. Serotype Asia1 occurs mainly in Asian regions. FMDV capsid protein VP1 is responsible for inducing a protective immune response. This study performs an in silico approach to determine antigenic heterogeneity within several antigenic sites of VP1. A total of 47 VP1 sequences from independent FMDV isolated from different countries of South Asian regions were selected and retrieved from the NCBI database. Sequences were aligned by EBI ClustalW program. In the absence of experimentally derived 3D structure data, the structure of VP1 protein of FMDV type Asia1 was modeled using a homology modeling approach. Several antigenic sites which may have a role in virus neutralization have been identified using both literature mining and computational predictive methods and then mapped onto the protein 3D structure. Variations at these antigenic sites were then analyzed by calculating the protein variability index. Amino acid substitutions at those sites were also identified and compared. It was found that antigenic variability that occurred during the past 12 years is not equally distributed at each VP1 antigenic site. Although several sites are more variable than other sites, interestingly for most antigenic sites, amino acid variability is lower than expected and fewer types of amino acid substitutions were evident. Three antigenic peptides have been identified as the most conserved epitopes for the past 12 years. This study revealed that although antigenic variability is high for FMDV for the past 12 years, only a few antigenic types have circulated in the South Asian region. This emphasizes a possibility to formulate improved synthetic vaccines for controlling foot-and-mouth disease in that region.
\end{abstract}

Key words: FMDV, VP1, homology modeling, antigenic variability. 